\title{
Stage IIB Mycosis Fungoides and Sezary Syndrome AJCC v8
}

National Cancer Institute

\section{Source}

National Cancer Institute. Stage IIB Mycosis Fungoides and Sezary Syndrome A/CC v8.

NCI Thesaurus. Code C141352.

Stage IIB includes: T3, N0-2, M0, B0,1. T3: One or more tumors (equal or greater than 1 cm in diameter). N0: No clinically abnormal peripheral lymph nodes; biopsy not required. N1: Clinically abnormal peripheral lymph nodes; histopathology Dutch grade 1 or National Cancer Institute (NCI) LN0-2. N2: Clinically abnormal peripheral lymph nodes; histopathology Dutch grade 2 or NCI LN3. MO: No visceral organ involvement. B0: Absence of significant blood involvement: $5 \%$ or less of peripheral blood lymphocytes are atypical (Sezary cells). B1: Low blood tumor burden: more than $5 \%$ of peripheral blood lymphocytes are atypical (Sezary cells) but does not meet the criteria of B2. (AJCC 8th ed.) 